\title{
Parents' Campaigns to Reduce their Children's Conduct Problems: Interactions with Temperamental Resistance to Control $^{*}$
}

\author{
Jackson A. Goodnight ${ }^{1}$, John E. Bates ${ }^{1}$, Gregory S. Pettit ${ }^{2}$, and Kenneth A. Dodge ${ }^{3}$ \\ ${ }^{1}$ Indiana University, USA \\ ${ }^{2}$ Auburn University, USA \\ ${ }^{3}$ Duke University, USA
}

\begin{abstract}
Longitudinal studies have found associations between parenting and the development of conduct problems, and have found that resistant to control temperament moderates these associations. Intervention studies have found associations between intervention-induced changes in parenting and subsequent reductions in children's conduct problems. However, no study to date has evaluated whether parents' self-initiated efforts to change their parenting practices affect children's conduct problems and whether effects depend on children's temperament. The current study asked whether parents' concerted efforts, or campaigns, to increase their involvement and limit-setting were effective in reducing growth in conduct problems from late childhood to early adolescence. It also asked whether the effects of campaigns varied according to children's levels of temperamental resistance to control. Analyses statistically controlled for parenting practices and conduct problems before the campaigns, socioeconomic status, gender, and ethnicity. Results indicated that campaigns that included increased involvement and limit-setting were beneficial only for youths who were rated in early childhood as temperamentally resistant to control.
\end{abstract}

\section{Keywords}

conduct problems; temperament; parenting; moderator effects; family change

Conduct problems show high levels of continuity from childhood to adolescence (Fergusson $\&$ Horwood, 1993). At the same time, individual trajectories of conduct problems show significant variability (Bongers, Koot, van der Ende, \& Verhulst, 2004). This suggests, given the very limited use of mental health services (Farmer, Stangl, Burns, Costello, \& Angold, 1999), that many children with early conduct problems show improvements in their behavior without the aid of professional intervention. How might such improvements occur? One possibility is that they are influenced by parents' deliberate, extended efforts to modify their child-management behaviors. This is a theoretically appealing possibility. We know of

\footnotetext{
*The Child Development Project has been funded by grants MH42498, MH57024, MH56961, and MH57095 from the National Institute of Mental Health and grant HD30572 from the National Institute of Child Health and Human Development. This work was also supported by National Research Service Award F31-MH076582-02 from the National Institute of Mental Health.

(C) Vandenhoeck \& Ruprecht GmbH \& Co. KG, Göttingen 2008

Address for correspondence: Jackson A. Goodnight, Department of Psychological and Brain Sciences, 1101 East Tenth Street, Indiana University, Bloomington, Indiana 47405. Phone: 812-855-1774. jgoodnig@ indiana.edu.
} 
anecdotal examples, but not of any empirical research that has evaluated the effects of these "campaigns" to reduce children's conduct problems.

Parenting has received perhaps more empirical attention than any other risk factor involved in the development of conduct problems. Researchers have demonstrated that parents who reinforce child demandingness and oppositionality are likely to see growth in these child characteristics, whereas parents who punish these behaviors and reinforce constructive conflict resolution behaviors are instead likely to see growth in prosocial behaviors (Patterson, Reid, \& Dishion, 1992; Snyder \& Patterson, 1995; Snyder, Schrepferman, \& St. Peter, 1997). The effects of parenting might transcend genetic influences. In an innovative, genetically-informed study, Caspi et al. (2004) found that differences in parental warmth and negativity directed toward identical twins predicted later differences in conduct problems between twins, even controlling for earlier conduct problems. The effects of parenting are also found after controlling for covarying risk factors. For example, in a longitudinal study utilizing multiple reporters and a community sample, Beyers, Bates, Pettit, and Dodge (2003) found that positive parenting, characterized by warmth, involvement, consistency, and monitoring of child behavior, predicted reduced growth in teacher-reported conduct problems in early adolescence when controlling for neighborhood characteristics.

Intervention research has provided additional evidence for the effects of parenting on conduct problem development. Studies show that parent-training interventions reduce child conduct problems (Dodge, Coie, \& Lynam, 2006). Schrepferman and Snyder (2002) found that the effectiveness of parenting-training intervention depended upon the degree to which improvements were observed in parenting behaviors. Children whose parents showed the largest increases in their reinforcement of nonaversive child behaviors relative to aversive child behaviors were at the lowest risk for arrest and out-of-home placement two years after treatment. Likewise, Beachaine, Webster-Stratton, and Reid (2005) found that children whose parents showed the greatest improvements in parenting during treatment (e.g., increased follow through on consequences for misbehavior) showed the largest declines in parent-reported conduct problems. Importantly, Beachaine et al. (2005) controlled for initial parenting behaviors, meaning that the effects of the intervention were attributable to changes, rather than pre-existing differences, in parenting.

Although intervention studies demonstrate that changes in parenting lead to reductions in existing conduct problems, they do not answer two other questions. They do not indicate whether the changes in parenting practices that yield improvements in conduct problems are the same aspects of parenting, that when deficient, lead to the initial development of conduct problems. And they do not indicate whether changes in parenting operate similarly outside of the context of a formal, professional intervention. In contrast, non-intervention longitudinal studies have shown that parenting practices predict the development of behavior problems, thus providing important information about the role of parenting in the natural developmental course of conduct problems. However, longitudinal studies have their own limitations. In general, longitudinal studies of parenting effects have not differentiated between stable and changing components of parenting (although we have begun to do so: see Pettit, Keiley, Laird, Bates, \& Dodge, 2007). They also have not differentiated between reactive and purposeful, goal-directed changes in parenting. As a result, they have not tested whether goal-directed changes in parenting are associated with reductions in conduct problems. We ask whether parents do in fact initiate attempts to change their children's behavior and whether they are effective. Considering that only a minority of parents experiencing child conduct problems will seek professional assistance (Farmer et al, 1999), and that many parents who eventually seek help are likely to have failed in their own attempts to reduce their children's conduct problems, parents' campaigns are an important area for scientific investigation. 
Our understanding of parenting effects is further complicated by findings that parenting does not predict conduct problems to the same extent for all children. Several parenting effects appear to depend on temperamental dimensions of resistance to control, negative emotionality, and fearfulness (Bates \& Pettit, 2006). Of most relevance to the current study are interactions between parenting and resistance to control and between parenting and fearlessness. Bates, Pettit, Dodge, and Ridge (1998) found that temperamental resistance to control moderated the effects of maternal control in childhood. Whereas a high level of maternal control predicted reduced conduct problems for temperamentally resistant children, it appeared to predict increased conduct problems for temperamentally nonresistant children. Bates et al. (1998) demonstrated this pattern in two independent samples (one of which was the sample for the present study), using a prospective design. In addition, their findings were evident using both parent and teacher reports of behavior problems. Kochanska (1997) found that positive, warm parent-child relations were most beneficial for temperamentally fearless young children, whereas gentle parental control was most beneficial for temperamentally fearful children. More specifically, fearless children who experienced a warm relationship with their mothers, and fearful children who experienced gentle control, showed the highest levels of internalization of rules during challenging laboratory tasks and the greatest numbers of prosocial responses to hypothetical moral dilemmas. Kochanska, Aksan, and Joy (2007) replicated these findings in two independent samples. In one sample, they found that a positive mother-child relationship (as assessed through observation) was more strongly predictive of future committed compliance with parental directives for children who showed little fear during a frightening laboratory procedure. In the other sample, they found that a positive mother-child relationship was more strongly predictive of children's self-rated moral development. Although Kochanska et al (2007) did not find this pattern when using measures of father-child positive relationship, it is still striking that mother-child relationship is consistently of special importance for fearless children.

A complementary pattern of interactions between parenting and impulsivity has been documented in adolescence. Goodnight et al. (2006) found that a composite measure of observed, parent-reported, and child reported positive parenting (e.g., positive reinforcement warmth, involvement) predicted lower levels of conduct problems for adolescents who were strongly attentive to rewards in a laboratory decision-making task, but not for adolescents who were not strongly attentive to rewards. This laboratory decision-making task, known as the Passive Avoidance or Go/No-Go Association Task, assessed individual differences in youths' tendency to focus on reward cues when learning the association between a series of numbers associated with monetary gains and losses (Yechiam et al., 2006). Goodnight et al (2006) used a prospective design in which earlier conduct problem levels were statistically controlled to eliminate the possibility that differences in the association between parenting and later conduct problems were attributable to pre-existing differences in conduct problems. Stice and Gonzalez (1998) found similar results in their analysis of parenting and conduct problems in adolescence. They found that a pattern of supportive and involved parenting was most strongly associated with low levels of conduct problems and drug use for boys who scored high in impulsivity. However, their study was limited by its cross-sectional design. Jones, Cauffman, and Piquero (2007) also reported similar findings. In an ethnically diverse sample of juvenile offenders, they found that supportive and involved parenting was more strongly associated with fewer conduct problems among youths who scored high on a questionnaire measure of impulsivity and risk-seeking. Supportive and involved parenting was strongly associated with low levels of conduct problems in youths who were high in impulsivity, but supportive parenting had no effect for youths who were low in impulsivity. This study was also limited by its cross-sectional design, and its sample limited the generalizability of its findings. Wright, Caspi, Moffitt, and Silva (2001) also reported findings that are highly consistent with this pattern of findings. They found that "family ties", which included measures of intimacy and involvement with parents during late adolescence, were more strongly predictive of low levels of criminal activity in 
early adulthood for youths low in self-control than for youths high in self-control. Self control was assessed using self, parent, teacher, and observer reports of impulsivity, hyperactivity, inattention, physical response to conflict, and risk-taking, and thus represented a very broadly defined measure of impulsivity. Regardless of differences in analytic approach and specific measure of impulsivity, these studies of parenting-temperament/personality interactions suggest that increased positive involvement and control are more strongly associated with reduced behavior problems for youths with impulsive characteristics than for youths without impulsive characteristics.

Explanations have been offered for the parenting-impulsivity interaction effects reviewed above. Hoffman (1983) described two pathways to socialization that may help explain why resistance to control/impulsivity moderates the effects of parenting. One pathway is based on anxiety conditioning. Children on this pathway learn to behave prosocially because they anticipate the negative consequences associated with misbehavior. A second pathway is based on an affectively positive and responsive relationship between parent and child. Children on this pathway behave prosocially because they feel motivated to accept their parent's goals and agendas. Kochanska (1997) argued that fearful children are more responsive to the anxietymediated pathway, and fearless children are more responsive to the positive relationship pathway. Other explanations are possible too. We have previously suggested that impulsive children may be especially responsive to positive parenting because they are more responsive to incentive-based learning opportunities than other youths (Goodnight et al., 2006). We have also suggested that resistant to control youths require firmer, more consistent control to offset their self-regulatory deficits (Bates et al., 1998). Along the same lines, Wright et al. (2001) argued that high self-control individuals are less strongly influenced by their social relationships than low self-control individuals, because high self-control individuals are better able to internally compensate for deficient prosocial social controls or excessive antisocial controls in their social groups. Although there has been a remarkable explosion of evidence that the association between parenting and child adjustment depends on the characteristics of the child, there has been no research, to our knowledge, on how child characteristics moderate the effects of parents' campaigns to improve child adjustment.

\section{The Current Study}

The current study evaluated the effects of parents' campaigns to reduce their children's conduct problems. This approach to studying parenting effects is different from past longitudinal research because it considers parents deliberate, extended efforts to reduce their children's conduct problems. As stated above, longitudinal studies of parenting effects have not distinguished this aspect of parenting from other aspects, such as stable elements and reactivitybased changes in parenting. The campaign approach is also different from intervention research because it considers parents attempts outside of the context of formal intervention. Often times in the context of formal interventions, parents receive in-depth training in various effective parenting behaviors. In contrast, the current approach asks whether parents are capable of making effective changes without this assistance.

The current study focused on two types of parenting, involvement and limit-setting, that past correlational and intervention research has shown to be associated with children's conduct problems. We hypothesized, based on the evidence reviewed above, that parents' campaigns to change their involvement and limit-setting would predict reductions in conduct problems. In addition, based on the same review, we hypothesized that parenting campaigns of these types would interact with child resistance to control. We expected that limit-setting and involvement campaigns would produce larger beneficial effects for temperamentally resistant to control children, who have shown greater benefits from control and positive parenting than have children who are not resistant to control. 
These hypotheses were tested by modeling individual variation in behavioral problem trajectories from age 10 to age 14, a period during which children show increased variability in behavior problems and a substantial number of parents were expected to report attempts to combat burgeoning conduct problems. The effects of campaigns were evaluated while statistically controlling for the potentially confounding effects of temperament, race, socioeconomic status, youth gender, and behavior problem levels prior to the start of parental campaigns. Importantly, the effects of pre-campaign (age 8) parenting behaviors were also included as a predictor in the model in order to evaluate the effects of campaigns over and above pre-existing differences in parenting.

\section{Method}

\section{Participants}

The Child Development Project (Dodge, Bates, \& Pettit, 1990) initially recruited 585 families from Nashville and Knoxville, TN, and Bloomington, IN. Participants were recruited at random during kindergarten preregistration in the spring prior to fall matriculation. About $15 \%$ of participants were recruited on the first day of school or by letter or telephone, in order to include families who did not preregister their children. Of those contacted, 75\% agreed to participate in the study. Eighty-one percent of the original sample were European-American, $17 \%$ were African-American, and 2\% were of other ethnicities. Families' Hollingshead (1979) socioeconomic status ranged from 8 to 66 , with a mean of $39.5(\mathrm{SD}=14.0)$.

\section{Measures}

Parents' Campaigns-As part of annual interviews when children were 11 years of age, participating parents (usually mothers) were asked whether they had made an extended effort in the past year or two to reduce their children's aggressive or rule breaking/disrespectful behaviors (regardless of the child's prior level of problem behaviors). Parents who stated that they had begun a campaign were asked to indicate what changes they made on a list of possibilities. Of interest to the current study were whether the parents endorsed "spen[ding] more time with their child" or "bec[oming) more strict or directive with their child." Of the 460 parents who participated when their children were 11 years of age, $16.1 \%$ reported mounting an involvement-focused campaign, and $19.6 \%$ reported mounting a limit-setting campaign. Of the 96 parents who reported either type of campaign, 68 reported limit-setting and involvement campaigns, 22 reported limit-setting campaigns without involvement campaigns, and 6 reported involvement campaigns without limit-setting campaigns. Thus, whereas limit-setting was a component of nearly of every campaign, involvement was an additional component of most, but not all, campaigns. Because so few parents reported involvement-only campaigns, direct comparisons between children who received involvement campaigns and those who received limit-setting campaigns could not be made. Instead, analyses focused on comparing the effects of a) limit-setting-only campaigns against no campaign, and b) limit-setting-plus-involvement campaigns against no campaign. Two binary variables were computed so these comparisons could be made. For the first variable, parents who reported becoming stricter or more directive (but did not report becoming more involved) were given a score of 1 , and parents who did not start a campaign were given a score of 0 . This variable was used in the first analysis to test the effect of limit-setting campaigns against no campaign. For the second variable, parents who reported spending more time with their children and becoming stricter or more directive were given a score of 1 , and parents who did not start a campaign were given a score of 0 . This variable was used in the second analysis to test the effect of involvement-plus-limit-setting campaigns against no campaign.

Although the campaign variables were based on single items, we believe they are face valid indicators of parents' beliefs regarding their attempts to reduce their children's problematic 
behavior. Providing indirect evidence for the validity of the campaign measures, Pettit et al. (2007) found that parents who rated their campaigns as effective showed less rapid declines in monitoring over time as compared to parents who did not rate their campaigns as effective. This finding suggests that parents' reports of campaigns are linked with changes in parenting practices over time.

Temperamental Resistance to Control-When children were five years of age, mothers completed the Retrospective Infant Characteristics Questionnaire (RICQ), a retrospective measure of infant temperament. Resistance to control, or unmanageability (Bates et al., 1998), was computed by averaging scores from four items measuring children's impulsive explorations and unresponsiveness to prohibitions (see Table 1 for items). This construct is believed to reflect an early form of impulsive personality that may stem from strong approach tendencies or weak inhibitory control (Bates et al., 1998). Individual items were rated on seven point scales. The resistance scale has shown adequate cross-age continuity from 13 to 24 months of age in another sample (Bates \& Bayles, 1984), and from 30 to 42 months of age in a small, ongoing pilot study of toddlers' self regulation ( $r$ f from .39 to .60 ). The resistance scale also has shown external validity in terms of its association with effortful control $(r=-$. 44 ) and anger/frustration (i.e., negative affect related to interruption of ongoing tasks; $r=.59$ ) scales of the Child Behavior Questionnaire (2000) in the pilot just mentioned, and more generally, with externalizing behaviors (Rothbart \& Bates, 1998). Other work has demonstrated continuity in temperament constructs from infancy through early childhood. Bates et al. (1998) reported a correlation of 34 between maternal report of resistance to control at 13-24 months of age and maternal retrospective report of resistance to control at 10 years of age. The scale used here had adequate internal consistency $(\alpha=.83)$.

Non-physical Child Management-When children were age 8, parents reported on how they corrected their children's misbehaviors. Seven of these questions were designated as nonphysical parenting practices on the basis of their consistency with parent training intervention content (e.g., Fleischman, Home, \& Arthur, 1983). These items included using timeout, denying privileges, instructing the child how to behave, explaining reasons or discussing, having child apologize, assigning extra chores, and giving rewards for good behavior. Parents rated themselves on the frequency with which they used each strategy on a five point scale, ranging from never (0) to about everyday (5). Scores on these items were averaged to create a composite measure of non-physical child management. The composite measure had adequate internal consistency $(\alpha=.81)$.

Demographics-Information on child race and sex was gathered from parent self-report at the beginning of the study (kindergarten). Family socioeconomic status (SES) was computed according to Hollingshead's (1979) procedure using information gathered from parent report at child age 11.

Conduct problems-Mothers completed the Child Behavior Checklist (Achenbach, 1991) annually from child age 10 to age 14. Items assessing delinquent and aggressive behaviors were summed according to Achenbach (1991) to create the externalizing problems scale. The empirically derived measure of externalizing behavior has shown adequate levels of reliability and validity.

\section{Data Analysis Plan}

Growth curve modeling was used to evaluate the effects of predictor variables on individual conduct problem trajectories. Individual trajectories were estimated using externalizing behavior problem data collected annually from age 10 to age 14 . The intercept of the growth curve was set at age 11 so that it corresponded with the timing of the campaign assessment. 
The intercept and slope parameters of the growth models were regressed on the predictor variables to determine the association between (1) behavior problem levels at age 11 (the intercept) and parents' decisions to begin a campaign, and between (2) behavior problem growth from age 10 until age 14 (the slope) and parents' decisions to begin a campaign. Resistance to control was centered by subtracting the sample mean from each individual's scores to reduce collinearity between resistance to control and the interaction between resistance to control and campaign, and to facilitate interpretation of the parameter estimates in the model. Interaction variables were calculated by multiplying the centered resistance to control scores with the dichotomous campaign variables. Mplus 4.2 (Muthén \& Muthén, 19982007) was used to estimate the models. Model one included 386 participants who either received a limit-setting campaign only or received no campaign at age 11. Model two included 432 participants who either received a limit-setting-plus-involvement campaign or received no campaign at age 11. Missing data for participants included in each analysis were addressed by using full information maximum likelihood (FIML) parameter estimation, which utilizes all available data to minimize the potential for biased parameter estimation. The FIML parameter estimation method has been shown to reduce bias relative to complete cases analysis and is likely to produce valid estimates even when data are not missing at random (Schafer \& Graham, 2002). A maximum likelihood estimator with robust standard errors (to account for effects of nonnormal distribution of behavior problems on standard errors) and nonnormal-adjusted fit indexes (including the Satorra-Bentler (SB) chi-square) were used to evaluate the fit of the models, because the externalizing scores were nonnormally distributed. Model fit was considered to be acceptable if Root Mean Squared Error of Approximation (RMSEA) was less than .08 (Browne \&Cudeck, 1993) and Comparative Fit Index (CFI) was greater than .90 (Bentler, 1992).

\section{Results}

\section{Preliminary Analyses}

Bivariate correlations between continuous study variables showed strong, positive associations between externalizing behaviors in all years. In addition, parents' non-physical management was positively associated with externalizing problems in all years. That is, as would be expected, parents who perceived higher levels of externalizing behavior problems reported using disciplinary tactics more frequently. SES was negatively associated with externalizing behaviors in all years and positively associated with non-physical management, and resistance to control was positively associated with externalizing behaviors in all years and non-physical management. SES and resistance to control were not associated with each other. See Table 2 for all correlations.

Chi-square tests revealed no significant associations between campaigns and race or between campaigns and sex, suggesting that campaigns were nearly equally distributed among boys and girls and among African American and Caucasian youths. In contrast, t-tests indicated higher levels of resistance to control and lower SES among youths who received involvementplus-limit-setting campaigns, and indicated higher levels of conduct problems for youths receiving either limit-setting-only or involvement-plus-limit-setting campaigns. See Table 3 for results from these analyses.

\section{Structural Equation Modeling}

Before testing hypotheses, two models (including all 460 participants who completed procedures at age 11) were compared in order to determine the best model for capturing conduct problem trajectories. The first model included two growth components, an intercept and slope, which captured linear growth over time. This model fit the data well: SB scaled $\chi^{2}(10, \mathrm{~N}=$ $460)=13.430 p=.20, \mathrm{CFI}=.992, \mathrm{RMSEA}=.027$. The second model included three growth 
components: an intercept, slope, and a quadratic parameter, which captured both linear and nonlinear growth. This model also fit the data well, but did not fit significantly better than the linear growth model (SB scaled $\Delta \chi^{2}(2)=4.69, p=.10$ ). Therefore, the linear growth model was chosen over the nonlinear growth model for use in testing the effects of predictor variables. The results of the linear growth model indicated that the mean of age 11 (intercept) conduct problems $(M=8.94)$ was significant, but average change (slope) in conduct problems from age 10 to $14(\mathrm{M}=.05)$ was nonsignificant. However, variances of intercepts and slopes were statistically significant (36.20 and 1.00, respectively, both $p<.05$ ), suggesting that there were meaningful differences between individuals in terms of their age 11 conduct problems and in terms of change in behavior problems from age 10 to 14. Finally, the correlation between intercept and slope was nonsignificant, meaning that youths who were high in conduct problems at age 11 did not change in their conduct problems at a rate that was different from youths who were low in conduct problems at age 11 .

After determining the model that best captured change in behavior problems from age 10 to 14 , hypotheses were tested by adding campaigns, resistance to control, interactions between campaigns and resistance to control, and control variables to the linear growth model specified above. The "limit-setting-only model" tested the effects of limit-setting-only campaigns, resistance to control, the interaction between limit-setting campaigns and resistance to control, gender, ethnicity, SES, and age 8 non-physical child management on age 11 (intercept) conduct problems and changes (slope) in conduct problems. See Figure 1 for a path diagram of this model. In addition, to control for the effects of conduct problems at the time of the start of the campaign on subsequent change in conduct problems, the intercept of conduct problems was used to predict the slope in conduct problems. The "involvement-plus-limit-setting model" included the same predictors except that the limit-setting-only campaign variable was replaced with the involvement-plus-limit-setting campaign variable in this model. See Figure 2 for a path diagram of this model.

Limit-Setting-Only Model-This model, which tested the effects of a limit-setting-only campaign against no campaign, fit the data adequately: $\mathrm{SB}$ scaled $\chi^{2}(31, \mathrm{~N}=386)=$ $67.633, p<.01, \mathrm{CFI}=.958$, RMSEA $=.055$. Limit-setting campaign $(b=4.21)$, resistance to control $(b=.98)$, and non-physical child management $(b=2.27)$ were all significantly and positively associated with conduct problems at age 11 (the intercept), indicating that children who received a limit-setting campaign, were high in resistance to control, and received high levels of non-physical child management at age 8 were high in conduct problems at age 11 . In addition, SES $(b=-0.07)$ was significantly and negatively associated with conduct problems at age 11, indicating that children in high SES families were low in conduct problems at age 11. See Figure 1 for standardized path coefficients.

Gender was significantly and positively associated with change in conduct problems $(b=0.34)$, indicating that, controlling for all other predictors, girls showed more growth in conduct problems from age 10 to 14 than boys. Neither limit-setting campaign nor any other variables were significant predictors of change in conduct problems, suggesting that limit-setting campaigns (without additional campaigns) did not result in statistically significant improvements in conduct problem during this period, regardless of level of resistance to control. See Figure 1 for standardized path coefficients from the limit-setting-only model.

Involvement-Plus-Limit-Setting Model-This model, which tested the effects of an involvement-plus-limit-setting campaign against no campaign, fit the data adequately: SB scaled $\chi^{2}(31, \mathrm{~N}=432)=68.290, \mathrm{p}<.01, \mathrm{CFI}=.967$, RMSEA $=.053$. Involvement-plus-limitsetting campaign $(b=5.77)$, resistance to control $(\mathrm{b}=1.00)$, the interaction between campaign and resistance to control $(b=1.32)$, and age 8 non-physical child management $(b=2.05)$ were all significantly and positively associated with conduct problems at age 11 , indicating that 
youths who received this type of campaign, who were high in resistance to control, or who received high levels of non-physical child management at age 8 were high in conduct problems at age 11. The significant interaction between campaign and resistance to control indicated that youths who received this type of campaign were especially likely to have high levels of conduct problems if they were also high in resistance to control, but not if they were low in resistance to control. The model also showed that SES $(b=-09)$ was significantly and negatively associated with age 11 conduct problems, indicating that children who were high in SES were low in conduct problems. Standardized coefficients are shown in Figure 2.

Considering predictors of change in conduct problems from age 10 to 14 , only the interaction between campaign and resistance to control $(b=-.66)$ was significantly associated with the slope of conduct problems. This association indicated that involvement-plus-limit-setting campaigns predicted reductions in conduct problems over time, but only for youths who were high in resistance to control. To better understand this finding, the effects of the campaign were computed at various levels of resistance control. For youths at $1 \mathrm{SD}$ below the mean of resistance to control, this campaign had an unstandardized effect of .37 $(p>.05)$ and a standardized effect of .13. For youths at the mean of resistance to control, this campaign had an unstandardized effect of $-.38(p>.05)$ and a standardized effect of -.13 , Finally, for youths $1 \mathrm{SD}$ above the mean in resistance to control, this campaign had an unstandardized effect of $-1.12(p<.01)$ and a standardized effect of -.38 . Further analysis indicated that the beneficial effect of this type of campaign was statistically significant for youths who were .5 SDs above the mean or higher in resistance to control. In other words, campaigns were beneficial only to those youths who were relatively high in temperamental resistance to control. See Figure 2 for all standardized path coefficients from this model, and see Figure 3 for conduct problem trajectories for youths low, medium, and high in resistance to control who did or did not receive a campaign.

\section{Discussion}

The current study tested whether two types of parental campaigns, one that included increasing limit-setting and another that included increasing both limit-setting and involvement, were associated with subsequent reductions in conduct problems. Analyses revealed that limitsetting-only campaigns were not associated with statistically significant reductions in conduct problems, regardless of youths' temperamental resistance to control in early childhood. In contrast, limit-setting-plus-involvement campaigns predicted statistically significant reductions in conduct problems, but only for youths who were at least half a standard deviation above the mean in resistance to control. These effects were evident despite controlling for the potentially confounding influences of gender, ethnicity, previous conduct problems, and previous parenting behaviors.

There are several possibilities why limit-setting-plus-involvement campaigns were most beneficial for temperamentally resistant children, whereas limit-setting-only campaigns were not. We interpret resistance to control as an early marker of impulsivity, which is often associated with high responsiveness to reward stimuli and/or poor inhibitory control. It is possible that temperamentally resistant children are highly susceptible to rewards provided by peers for antisocial behavior and/or are unresponsive to typical punishments for antisocial behavior (Goodnight, Bates, Newman, Dodge, \& Pettit, 2006). To the degree that these pathways to conduct problems operate, campaigns that simply involve increasing limits on such children may not be sufficient, because they do not address the dominant motivations of such youths. However, increased limit-setting in combination with increased involvement between parent and youth may activate these youths' reward-seeking motives and provide effective incentives for more prosocial and less antisocial behavior. From a slightly different perspective, it is also possible that increased involvement contributed to an affectively-positive 
relationship between parents and youths that enhanced temperamentally resistant to control and reward-sensitive youths' eagerness to behave consistent with parental expectations. Yet another potential explanation is that through increased involvement parents became more consistent in monitoring their youths' behavior, and thus were able to help their resistant to control children avoid situations that facilitated or rewarded antisocial behavior. Given the elevated risk that resistant to control children have for conduct problems, further research is needed to identify the specific mechanisms at work in effective campaigns. It is possible that more than one of the mechanisms may operate in a single case, or that different mechanisms may explain different cases.

The lack of a significant effect of limit-setting campaigns for children who were high in resistance to control may appear to be inconsistent with Bates et al. (1998), which found that reactive parental control in early childhood was associated with reductions in conduct problems exclusively for children who were rated as being high in resistance to control. There are several possible explanations for this apparent inconsistency. First, it is likely that the young children's parents who were high in reactive control in Bates et al, (1998) were also high in their involvement. These parents were responding frequently to their children's minor misbehaviors, and this implies close attention, which is a form of involvement. Even in the preadolescent children's parents' choice of campaigns, parents who started a campaign were more likely to increase both limit-setting and involvement than to increase just one or the other. If it is true that parents who were high in reactive control were also high in involvement, it would be more appropriate to compare the Bates et al. (1998) finding to the finding regarding the effects of campaigns that included limit-setting and involvement efforts. The effects of this dual-effort campaign were similar to the finding in Bates et al., in that temperamentally resistant children were more responsive than other children to parents' efforts. If it is not true that the early reactive control was paired with involvement, a second interpretation is that differences in findings between Bates et al, (1998) and the effects of limit-setting-only campaigns may stem from differences in the developmental periods being considered in the two studies. Perhaps limit-setting-only may be sufficient for children in early childhood (the period considered by Bates et al.), but limit-setting without additional efforts may be insufficient for youths in the transition to adolescence, where they are more independent and socially active than in early childhood. Parents may not have frequent enough contact with their resistant to control adolescents to effect change simply by increasing limits. Instead, they may have to encourage prosocial behavior by also increasing their involvement with their children.

It is challenging to explain why children who were moderate or low in resistance to control did not benefit from parental campaigns. Perhaps these differences in effects for children high versus low in resistance to control could be explained in part by differences in the causes of conduct problems for youths who are resistant to control and youths who are not. Although it has been argued that impulsive youths' conduct problems are the product of self-regulatory deficits or sensitivity to reward and punishment cues (Newman \& Lorenz, 2002), nonimpulsive youths' conduct problems could be the product of other factors, such as social information processing deficits and longstanding deficiencies in parental socialization efforts. If this is the case, typical parenting campaigns that emphasize consequences for behavior may not be as beneficial for nonimpulsive youths as parenting campaigns that feature the teaching of social skills or accurate attributions of intent in social situations.

Another possible explanation comes from previous analyses of parenting using this dataset. Pettit and Laird (2002) found that proactive parental involvement in response to high levels of child behavior problems predicted improved monitoring, but that proactive parental involvement in response to low levels of child behavior problems predicted increased psychological control. These findings suggest that campaigns for low resistant children, who show relatively few behavior problems, could lead to controlling or intrusive parenting 
practices that either fail to dampen or perhaps even contribute to increased conduct problems. Supporting this possibility, Pettit et al. (2007) found that parental campaigns predicted reductions in effective parenting practices in some families. Replications and extensions of the current study must be conducted before these potential explanations can be evaluated. In addition, this research suggests the need to understand distinct causal pathways for the development of conduct problems within subgroups of children with different temperament characteristics.

\section{Parental Motivations to use Campaigns}

Parents' report of having initiated a campaign was found to be associated with several control variables. Low SES and temperamental resistance to control increased the likelihood of using a limit-setting-plus-involvement campaign, but did not increase likelihood of a limit-settingonly campaign. However, high levels of non-physical child management increased the likelihood of reporting either campaign. These findings might mean that the control variables of SES, resistance to control, and previous parental efforts to manage child misbehaviors influence parents' decisions to start a campaign. Moreover, it is possible that some of these influences on parents' use of campaigns could be mediated by their effects on conduct problems. For example, temperamental resistance to control could be associated with increased conduct problems that are in turn associated with an increased likelihood of beginning a campaign. Alternatively, it is possible that the associations do not reflect a developmental process, but rather can be explained by the control variables' and campaign variables' shared associations with conduct problem levels. For example, it is possible that frequent use of child management behaviors and the decision to start a campaign are both due to having a child with high levels of conduct problems. Further research will be required to resolve these possibilities.

\section{Strengths and Limitations}

The current study had a number of strengths. It utilized a moderately large, socio-economically diverse, community sample. It also used longitudinal data and several statistical controls to reduce the possibility that the effects of campaigns were due to preexisting differences between families who began campaigns and those who did not. Furthermore, the inclusion of precampaign parenting in the analyses meant that the effects attributable to campaigns were due to changes associated with campaigns, not to preexisting differences in parenting that were associated with campaigns. In addition, the effect of the limit-setting and involvement campaign on subsequent change in conduct problems for resistant to control youths may be generalizable to youths with high levels of conduct problems. Figure 3 shows the estimated conduct problem trajectories for youths who did or did not receive campaigns, further divided for different levels of resistance to control. The resistant to control youths who received involvement-plus-limit-setting campaigns scored in the borderline clinical range (16-19 for males and 12-15 for females; Achenbach, 1991) on the CBCL at age 10. However, by age 14, these youths were likely to score below the clinical range (but not at levels of children who did not receive campaigns). Although clinical cutoffs may be arbitrary when considering small changes in conduct problems, the findings nonetheless suggest that parental campaigns may disrupt continuity in conduct problems for youths showing clinically significant levels of behavior problems.

The current study had a number of limitations as well. Parents reported on all variables. Thus, the size of some of the effects could have been biased upwards. However, it is difficult to imagine how shared method variance could account for the interaction effect. The current study was also limited by the fact that only a few youths received an involvement campaign without additional limit-setting, thus making it impossible to test the effects of an involvement-only campaign. In addition, sample sizes were too small to make direct comparisons between the two types of campaigns. As a result, the analyses were limited to testing how a typical limit- 
setting campaign fared with and without the addition of increased involvement. The study was also limited by the effects of the small size of the limit-setting-only group on statistical power. It is quite possible that, given a larger sample size, the small main effect of limit-setting campaigns (standardized effect of -.17) would have been significant. Finally, the current study was also limited by its measures of campaigns. It was not possible in the current study to test whether parents' reports of campaigns were associated with other indicators of changes in parenting. More detailed, dimensional measures of campaigns would have provided additional opportunities for examining how and for whom campaigns were effective. In addition, determining the specific changes in parenting that took place during the campaigns would be useful for development scientists and practitioners alike.

In conclusion, the current study showed that parental campaigns have the potential to reduce conduct problems in children who show early resistance to control. However, it also suggests that limit-setting-plus-involvement campaigns may be ineffective for youths who are average or low in resistance to control. Given the limitations of this study, especially in terms of measurement of campaigns and small group sizes, the findings should be interpreted with caution. Further research on the topic of parenting campaigns is required before specific recommendations can be made to parents in regards to efforts to reduce their children's conduct problems. Nonetheless, the current study takes an initial step in filling a gap in our understanding of how parents influence children's conduct problem development. If future research successfully replicates these findings and identifies more clearly the specific mechanisms at work, it may become possible to make temperamentally-tailored recommendations to parents that improve the effectiveness of their efforts to reduce their children's conduct problems.

\section{References}

Achenbach, TM. Manual for the Child Behavior Checklist/4-18 and 1991 Profile. Burlington, VT: University of Vermont Department of Psychiatry; 1991.

Bates JE, Bayles K. Objective and subjective components in mothers' perceptions of their children from age 6 months to 3 years. Merrill-Palmer Quarterly 1984;30:111-130.

Bates, JE.; Pettit, GS. Temperament, parenting, and socialization. In: Grusec, J.; Hastings, P., editors. Handbook of Socialization. New York: Guilford; 2006. p. 153-181.

Bates JE, Pettit GS, Dodge KA, Ridge B. Interaction of temperamental resistance to control and restrictive parenting in the development of externalizing behavior. Developmental Psychology 1998;34:982-995. [PubMed: 9779744]

Beauchaine TP, Webster-Stratton C, Reid MJ. Mediators, moderators, and predictors of 1-year outcomes among children treated for early-onset conduct problems: A latent growth curve analysis. Journal of Consulting and Clinical Psychology 2005;73:371-388. [PubMed: 15982136]

Bentler PM. On the fit of models to covariances. Psychological Bulletin 1992;112:400-404. [PubMed: 1438635]

Beyers JM, Bates JE, Pettit GS, Dodge KA. Neighborhood structure, parenting processes, and the development of youths' externalizing behaviors: A multilevel analysis. American Journal of Community Psychology 2003;31:35-53. [PubMed: 12741688]

Bongers IL, Koot HM, van der Ende J, Verhulst FC. Developmental trajectories of externalizing behaviors in childhood and adolescence. Child Development 2004;75:1523-1537. [PubMed: 15369529]

Browne, MW.; Cudeck, R. Alternative ways of assessing model fit. In: Bollen, KA.; Long, JS., editors. Testing structural equation models. Nembury Park, CA: Sage; 1993.

Caspi A, Moffitt TE, Morgan J, Rutter M, Taylor A, Arseneault L, et al. Maternal expressed emotion predicts children's antisocial behavior problems: Using monozygotic-twin differences to identify environmental effects on behavioral development. Developmental Psychology 2004;40:149-161. [PubMed: 14979757] 
Dodge KA, Bates JE, Pettit GS. Mechanisms in the cycle of violence. Science 1990;250:1678-1683. [PubMed: 2270481]

Dodge, KA.; Coie, JD.; Lynam, DR. Aggression and antisocial behavior in youth. In: Eisenberg, N.; Damon, W.; Lerner, RM., editors. Handbook of child psychology: Social, emotional, and personality development. Vol. 6 ed.. Vol. Vol. 3. Hoboken, NJ: John Wiley \& Sons Inc.; 2006. p. 719-788.

Farmer EMZ, Stangl DK, Burns BJ, Costello EJ, Angold A. Use, persistence, and intensity: Patterns of care for children's mental health across one year. Community Mental Health Journal 1999;35:3146. [PubMed: 10094508]

Fergusson DM, Horwood LJ. The structure, stability and correlations of the trait components of conduct disorder, attention deficit and anxiety/withdrawal reports. Journal of Child Psychology and Psychiatry 1993;34:749-766. [PubMed: 8340443]

Fleischman, MJ.; Horne, AM.; Arthur, JL. Troubled families: A treatment program. Champaign, IL: Research Press Company; 1983.

Goodnight, JA.; Bates, JE.; Kuwabara, M.; Newman, JP.; Pettit, GS.; Dodge, KA. Positive parenting and adolescent adjustment: The moderating effect of reward sensitivity; Paper presented at the biennial meeting of the Society for Research in Adolescence; 2006.

Goodnight JA, Bates JE, Newman JP, Dodge KA, Pettit GS. The interactive influences of friend deviance and reward dominance on the development of externalizing behavior during middle adolescence. Journal of Abnormal Child Psychology 2006;34:573-583. [PubMed: 16823636]

Hoffman, ML. Affective and cognitive processes in moral internalization. In: Higgins, ET.; Ruble, D.; Hartup, W., editors. Social cognition and social development: A socio-cultural perspective. New York: Cambridge University Press; 1983. p. 236-274.

Hollingshead, AA. Four-factor index of social status. New Haven, CT: Yale University; 1979. Unpublished manuscript

Jones S, Cauffman E, Piquero AR. The influence of parental support among incarcerated adolescent offenders: The moderating effects of self-control. Criminal Justice and Behavior 2007;34:229-245.

Kochanska G. Multiple pathways to conscience for children with different temperaments: From toddlerhood to age 5. Developmental Psychology 1997;33:228-240. [PubMed: 9147832]

Kochanska G, Aksan N, Joy ME. Children's fearfulness as a moderator of parenting in early socialization: Two longitudinal studies. Developmental Psychology 2007;43:222-237. [PubMed: 17201521]

Muthén, LK.; Muthén, BO. Mphs user's guide. Los Angeles: Muthén \& Muthén; 1998-2007.

Newman, JP.; Lorenz, A. Response modulation and emotion processing: Implications for psychopathy and other dysregulatory psychopathology. In: Davidson, RJ.; Scherer, K.; Goldsmith, HH., editors. Handbook of affective sciences. Oxford University Press; 2002. p. 1043-1067.

Patterson, GR.; Reid, JB.; Dishion, TJ. Antisocial boys. Eugene, OR: Castalia; 1992.

Pettit GS, Keiley MK, Laird RD, Bates JE, Dodge KA. Predicting the developmental course of motherreported monitoring across childhood and adolescence from early proactive parenting, child temperament, and parents' worries. Journal of Family Psychology 2007;21:206-217. [PubMed: 17605543]

Pettit, GS.; Laird, RD. Psychological control and monitoring in early adolescence: The role of parental involvement and earlier child adjustment. In: Barber, BK., editor. Intrusive parenting: How psychological control affects children and adolescents. Washington, D. C.: American Psychological Association; 2002. p. 97-123.

Rothbart MK, Ahadi SA, Evans DE. Temperament and personality: Origins and outcomes. Journal of Personality and Social Psychology 2000;78:122-135. [PubMed: 10653510]

Rothbart, MK.; Bates, JE. Temperament. In: Damon, W.; Eisenberg, N., editors. Handbook of child psychology. Vol. 5 ed.. Vol. Vol. 3. New York: Wiley; 1998. p. 105-176.

Schafer JL, Graham JW. Missing data: Our view of the state of the art. Psychological Methods 2002;7:147-177. [PubMed: 12090408]

Schrepferman L, Snyder J. Coercion: The link between treatment mechanisms in behavioral parent training and risk reduction in child antisocial behavior. Behavior Therapy 2002;33:339-359.

Snyder J, Patterson GR. Individual differences in social aggression: A test of a reinforcement model of socialization in the natural environment. Behavior Therapy 1995;26:371-391. 
Snyder J, Schrepferman L, St. Peter C. Origins of antisocial behavior: Negative reinforcement and affect dysregulation of behavior as socialization mechanisms in family interaction. Behavior Modification 1997;21:187-215. [PubMed: 9086866]

Stice E, Gonzales N. Adolescent temperament moderates the relation of parenting to antisocial behavior and substance use. Journal of Adolescent Research 1998;13:5-31.

Wright BRE, Caspi A, Moffitt TE, Silva P. The effects of social ties on crime vary by criminal propensity: A life-course model of interdependence. Criminology 2001;39:321-352.

Yechiam E, Goodnight JA, Bates JE, Busemeyer JR, Dodge KA, Pettit GS, et al. A formal cognitive model of the go/no-go discrimination task: Evaluation and implications. Psychological Assessment 2006;18:239-249. [PubMed: 16953727]

\section{Biographies}

Jackson A. Goodnight is a graduate student in the Department of Psychological and Brain Sciences at Indiana University-Bloomington. His research focuses on the interactive effects of self-regulation and social experiences on the development of conduct problems in childhood and adolescence.

John B. Bates is Professor of Psychological and Brain Sciences at Indiana UniversityBloomington. His research considers the development of behavior problems, with special emphasis on the interaction of individual characteristics, such as temperament, and environmental characteristics, such as parenting.

Gregory S. Pettit is a professor in the Department of Human Development and Family Studies at Auburn University. His research focuses on the mechanisms through which social experiences exert an impact on important developmental outcomes in childhood and adolescence and on the risk and protective factors that moderate these linkages.

Kenneth A. Dodge is the William McDougall Professor of Public Policy and Psychology and Neuroscience at Duke University, where he directs the Center for Child and Family Policy. His research addresses the development, prevention, and public policy of violent behavior. 


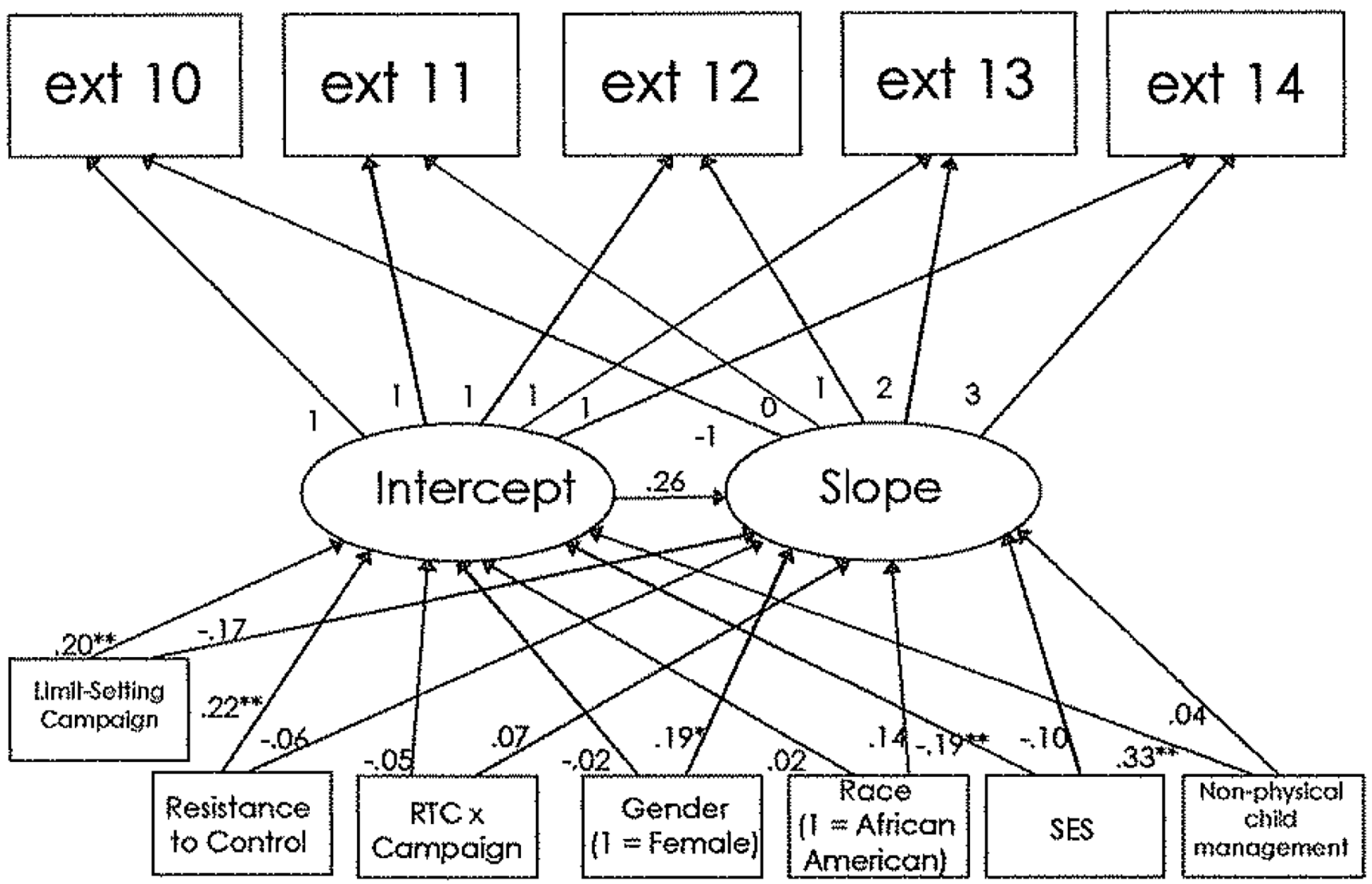

Figure 1.

Graphical representation of the limit-setting-only campaign model including predictors.

Parameter estimates are standardized. Ext $=$ externalizing. $\mathrm{RTC}=$ resistance to control. $*=p$ $<.05, * *=p<.01$. 


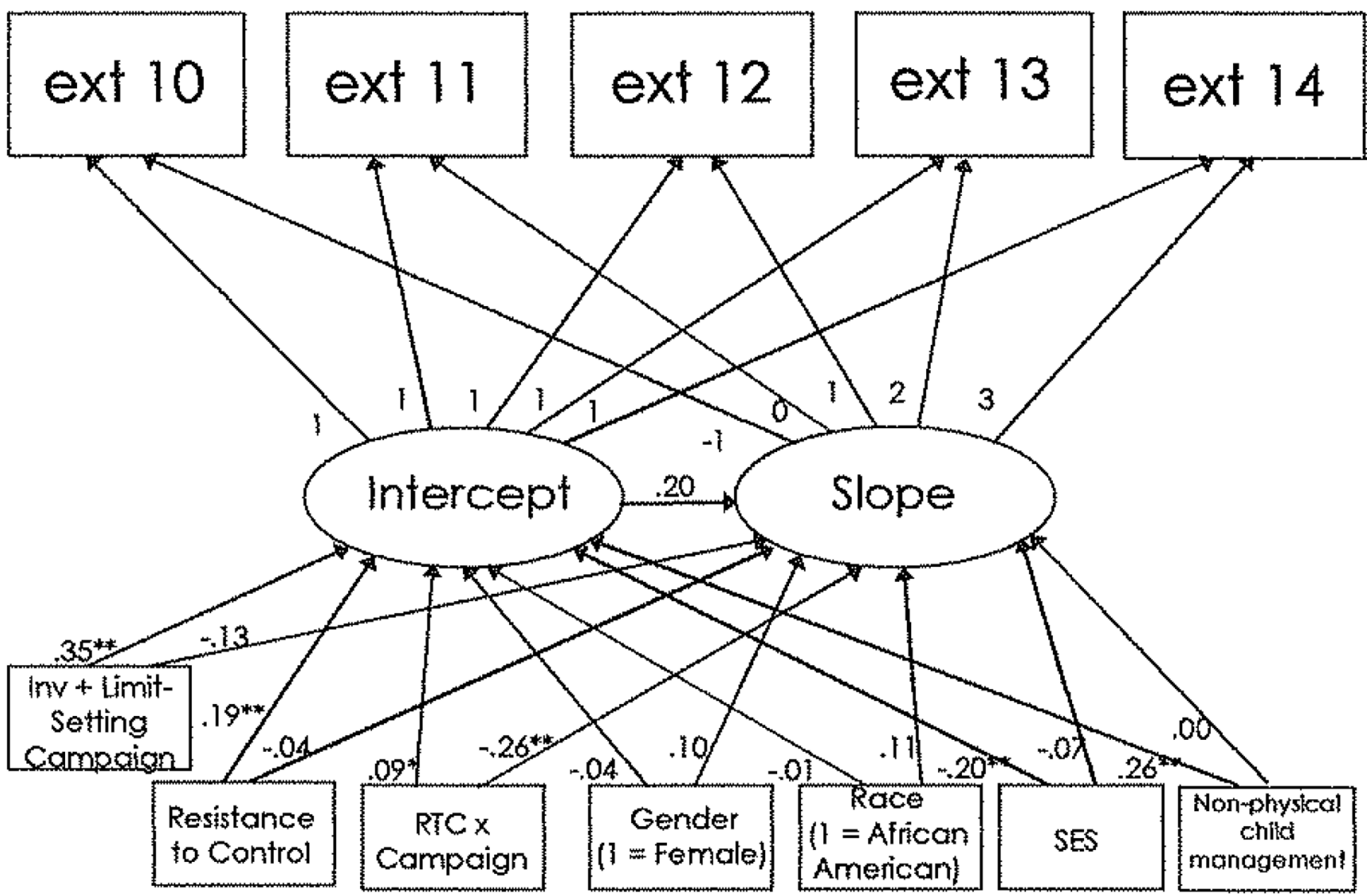

Figure 2.

Graphical representation of the involvement-plus-limit-setting campaign model including predictors. Parameter estimates are standardized. Ext $=$ externalizing. $\mathrm{RTC}=$ resistance to control. $*=p<.05, * *=p<.01$. 


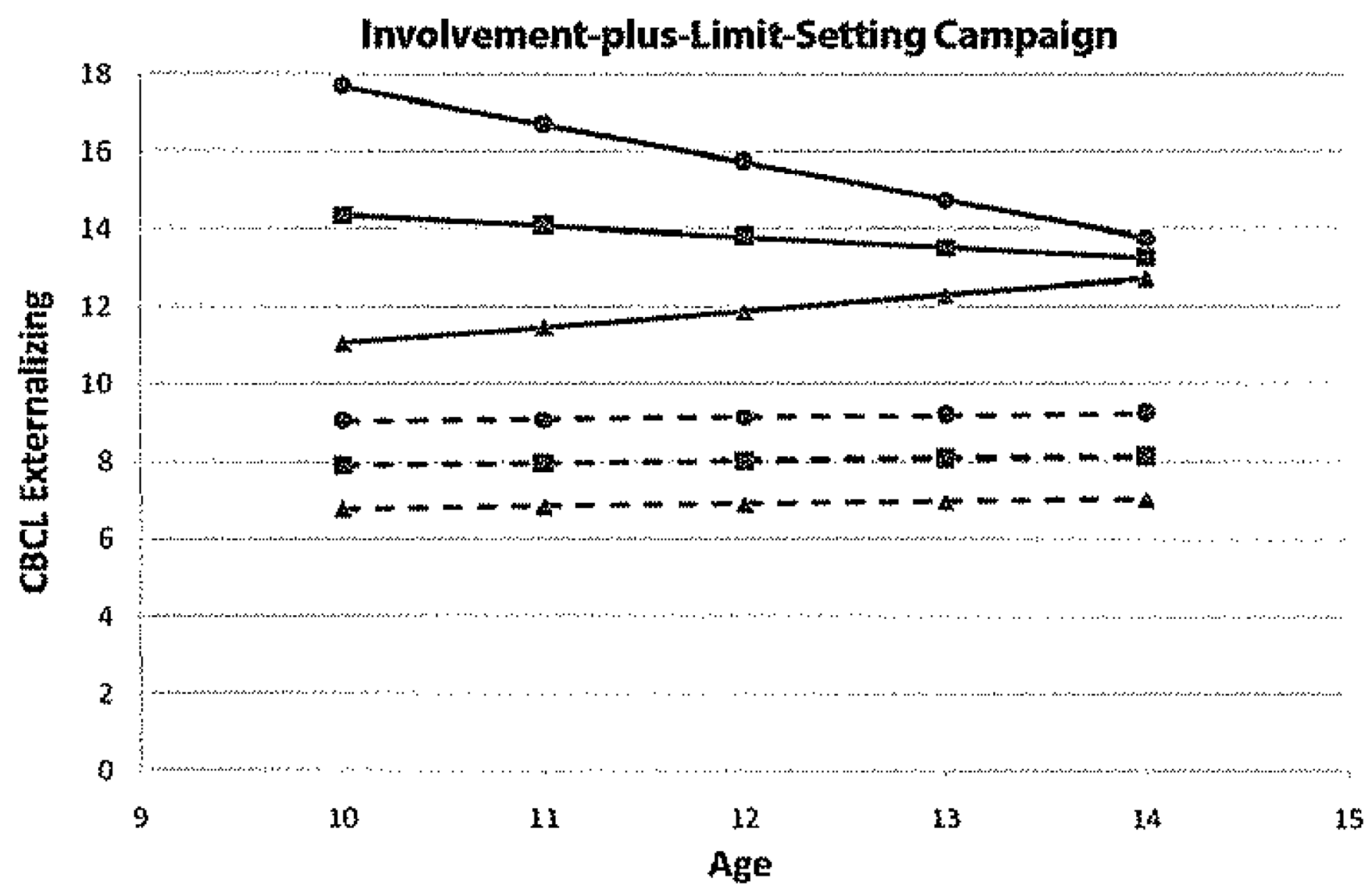

Figure 3.

Model-implied externalizing behavior trajectories for youths not receiving involvement-pluslimit-setting campaigns and for youths receiving involvement-plus-limit-setting campaigns who were rated as either low, medium, or high in resistance to control. RTC $=$ resistance to control. 


\section{Table 1}

Items from Resistance to Control Scale of the R-ICQ.

\begin{tabular}{l}
\hline 1. Persisted in playing with objects when told to leave them alone. \\
2. Continued to go someplace even when told "stop," "come here," "no-no". \\
3 Upset when removed from something she or he was interested in but should not be getting into. \\
$4 . \quad$ How much cuddle and snuggle when held (scaled from a lot to very little, seldom snuggles). \\
\hline
\end{tabular}




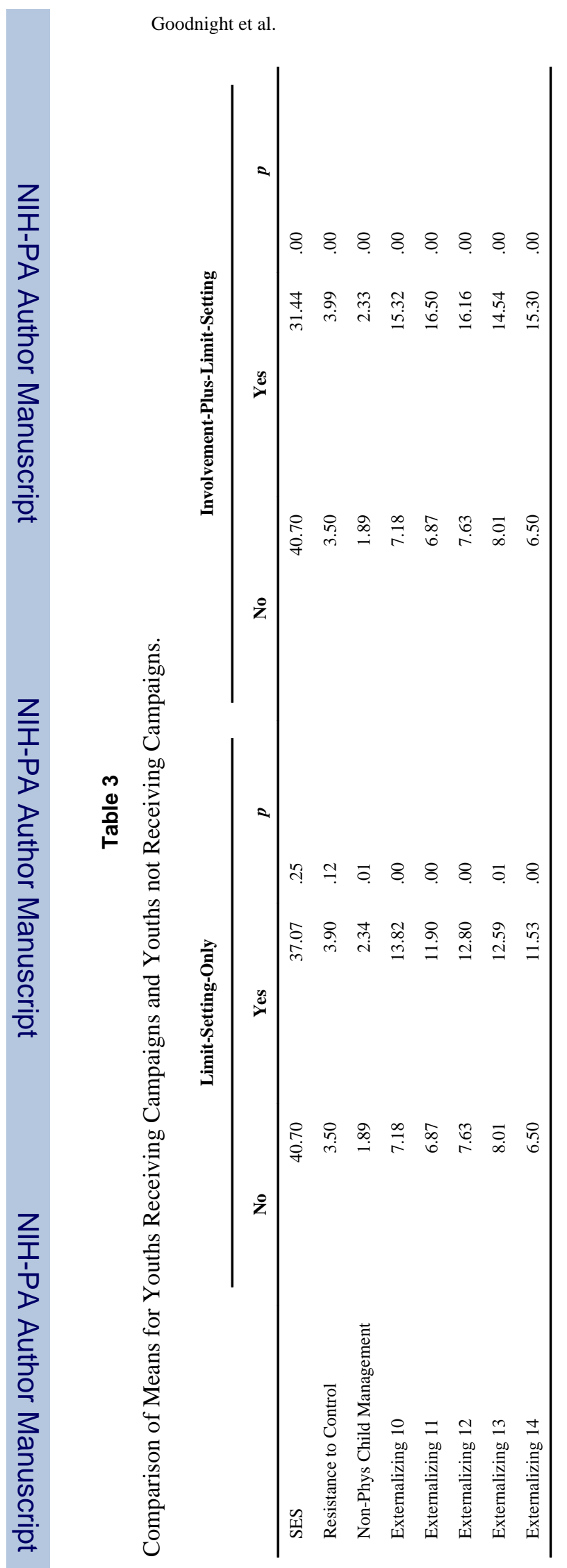

Page 20 